\title{
Signed (b,k)-Edge Covers in Graphs
}

\author{
A. N. Ghameshlou ${ }^{1}$, Abdollah Khodkar ${ }^{2}$, R. Saei ${ }^{3}$, S. M. Sheikholeslami*3 \\ ${ }^{1}$ Department of Mathematics, University of Mazandaran Babolsar, I.R., Iran \\ ${ }^{2}$ Department of Mathematics, University of West Georgia, Carrollton, USA \\ ${ }^{3}$ Department of Mathematics, Azarbaijan University of Tarbiat MoallemTabriz, I.R., Iran \\ Email:akhodkar@westga.edu,s.m.sheikholeslami@azaruniv.edu
}

\begin{abstract}
Let $G$ be a simple graph with vertex set $V(G)$ and edge set $E(G)$. Let $G$ have at least $k$ vertices of degree at least $b$, where $k$ and $b$ are positive integers. A function $f: E(G) \rightarrow\{-1,1\}$ is said to be a signed $(b, k)$-edge cover of $G$ if $\sum_{e \in E(v)} f(e) \geq b$ for at least $k$ vertices $v$ of $G$, where $E(v)=\{u v \in E(G) \mid u \in N(v)\}$. The value $\min \sum_{e \in E(G)} f(e)$, taking over all signed $(b, k)$-edge covers $f$ of $G$ is called the signed $(b, k)$-edge cover number of $G$ and denoted by $\rho_{b, k}^{\prime}(G)$. In this paper we give some bounds on the signed $(b, k)$-edge cover number of graphs.
\end{abstract}

Keywords: Signed Star Dominating Function, Signed Star Domination Number, Signed $(b, k)$-edge Cover, Signed $(b, k)$-edge Cover Number

\section{Introduction}

Structural and algorithmic aspects of covering vertices by edges have been extensively studied in graph theory. An edge cover of a graph $\mathrm{G}$ is a set $\mathrm{C}$ of edges of $\mathrm{G}$ such that each vertex of $\mathrm{G}$ is incident to at least one edge of $\mathrm{C}$. Let $\mathrm{b}$ be a fixed positive integer. A b-edge cover of a graph $\mathrm{G}$ is a set $\mathrm{C}$ of edges of $\mathrm{G}$ such that each vertex of $\mathrm{G}$ is incident to at least $\mathrm{b}$ edges of $\mathrm{C}$. Note that a $b$-edge cover of $\mathrm{G}$ corresponds to a spanning subgraph of $\mathrm{G}$ with minimum degree at least $b$. Edge covers of bipartite graphs were studied by König [1] and Rado [2], and of general graphs by Gallai [3] and Norman and Rabin [4], and $b$-edge covers were studied by Gallai [3]. For an excellent survey of results on edge covers and $b$-edge covers, see Schrijver [5].

We consider a variant of the standard edge cover problem. Let $G$ be a graph with vertex set $V(G)$ and edge set $E(G)$. We use [6] for terminology and notation which are not defined here and consider only simple graphs without isolated vertices. For every nonempty subset $E^{\prime}$ of $E(G)$, the subgraph of $G$ whose vertex set is the set of vertices of the edges in $E^{\prime}$ and whose

*Corresponding author edge set is $E^{\prime}$, is called the subgraph of $G$ induced by $E^{\prime}$ and denoted by $G\left[E^{\prime}\right]$. Two edges $e_{1}, e_{2}$ of $G$ are called adjacent if they are distinct and have a common vertex. The open neighborhood $N_{G}(e)$ of an edge $e \in E(G)$ is the set of all edges adjacent to $e$. Its closed neighborhood is $N_{G}[e]=N_{G}(e) \cup\{e\}$. For a function $f: E(G) \rightarrow \mathrm{R}$ and a subset $S$ of $E(G)$ we define $f(S)=\sum_{e \in S} f(e)$. The edge-neighborhood $E_{G}(v)$ of a vertex $v \in V(G)$ is the set of all edges incident to vertex $v$. For each vertex $v \in V(G)$, we also define $f(v)=\sum_{e \in E_{G}(v)} f(e)$. Let $b$ be a positive integer and let $G$ have at least $k$ vertices of degree at least $b$. A function $f: E(G) \rightarrow\{-1,1\}$ is called a signed $(b, k)$-edge cover (SbkEC) of $G$, if $f(v) \geq b$ for at least $k$ vertices $v$ of $G$. The signed $(b, k)$-edge cover number of a graph $\mathrm{G}$ is $\rho_{b, k}^{\prime}(G)=\min \left\{\sum_{e \in E} f(e) \mid f\right.$ is an SbkEC on $\left.G\right\}$. The signed $(b, k)$-edge cover $f$ of $G$ with $f(E(G))=\rho_{b, k}^{\prime}(G)$ is called a $\rho_{b, k}^{\prime}(G)$-cover. For any signed $(b, k)$-edge cover $f$ of $G$ we define 
$P=\{e \in E \mid f(e)=1\} \quad, \quad M=\{e \in E \mid f(e)=-1\}$,

$V^{+}=\{v \in V \mid f(v) \geq b\}$ and $V^{-}=\{v \in V \mid f(v)<b\}$.

If $b=1$ and $k=n$, then the signed $(b, k)$-edge cover number is called the signed star domination number. The signed star domination number was introduced by $\mathrm{Xu}$ in [7] and denoted by $\gamma_{S S}{ }^{\prime}(G)$. The signed star domination number has been studied by several authors (see for example [7,10]).

If $b=1$ and $1 \leq k \leq n$, then the signed $(b, k)$-edge cover number is called the signed star $k$-subdomination number. The signed star $k$-subdomination number was introduced by Saei and Sheikholeslami in [11] and denoted by $\gamma_{S S}^{k}(G)$.

If $b$ is an arbitrary positive integer and $k=n$, then the signed $(b, k)$-edge cover number is called the signed $b$-edge cover number. The signed $b$-edge cover number was introduced by Bonato et al. in [12] and denoted by $\rho_{b}^{\prime}(G)$.

The purpose of this paper is to initiate the study of the signed $(b, k)$-edge cover number $\rho_{b, k}^{\prime}(G)$. Here are some well-known results on $\gamma_{S S}{ }^{\prime}(G), \quad \gamma_{S S}^{k}(G)$ and $\rho_{b}^{\prime}(G)$.

Theorem 1 [10] For every graph $\mathrm{G}$ of order $n \geq 4$, $\rho_{1, n}{ }^{\prime}(G) \leq 2 n-4$.

Theorem 2 [11] For every graph $G$ of order $n \geq 4$ without isolated vertices, $\rho_{1, k}{ }^{\prime}(G) \leq n+k-4$.

Theorem 3 [10] For every graph $G$ of order $n$ without isolated vertices, $\rho_{1, n}{ }^{\prime}(G) \geq\left\lceil\frac{n}{2}\right\rceil$.

Theorem 4 [11] For every graph $G$ of order $n \geq 2$ without isolated vertices,

$$
\rho_{1, k}^{\prime}(G) \geq\left\lceil\frac{(\Delta(G)+1) k-n \Delta(G)}{2}\right\rceil .
$$

Theorem 5 [12] Let $b$ be a positive integer. For every graph $G$ of order $n$ and minimum degree at least $b$,

$$
\rho_{b, n}{ }^{\prime}(G) \geq\left\lceil\frac{b n}{2}\right\rceil .
$$

We make use of the following result in this paper.

Theorem 6 [7] Every graph $G$ with $\delta(G) \geq 3$ contains an even cycle.

\section{Lower Bounds for SbkECN of Graphs}

In this section we present some lower bounds on $\rho_{b, k}^{\prime}$ in terms of the order, the size, the maximum degree and the degree sequence of $G$. Our first proposition is a generalization of Theorems 3,4 and 5 .

Proposition 1 Let $G$ be a graph of order $n$ without isolated vertices and maximum degree $\Delta=\Delta(G)$. Let $b$ be a positive integer and let $n_{0} \geq 1$ be the number of vertices with degree at least $b$. Then for every positive integer $1 \leq k \leq n_{0}$,

$$
\rho_{b, k}^{\prime}(G) \geq \frac{k(b+\Delta)-n_{0}(\Delta-b+1)-n(b-1)}{2} .
$$

Proof. Let $f$ be a $\rho_{b, k}^{\prime}(G)$-cover. We have

$$
\begin{aligned}
\rho_{b, k}^{\prime}(G) & =\sum_{e \in E(G)} f(e)=\frac{1}{2} \sum_{v \in V(G)} \sum_{e \in E(v)} f(e) \\
& =\frac{1}{2} \sum_{v \in V^{+}} \sum_{e \in E(v)} f(e)+\frac{1}{2} \sum_{v \in V^{-}} \sum_{e \in E(v)} f(e) \\
& \geq \frac{k b}{2}-\frac{\left(n_{0}-k\right) \Delta+\left(n-n_{0}\right)(b-1)}{2} \\
& =\frac{k(b+\Delta)-n_{0}(\Delta-b+1)-n(b-1)}{2} .
\end{aligned}
$$

Theorem 2 Let $G$ be a graph of order $n$, size $m$, without isolated vertices and with degree sequence $\left(d_{1}, d_{2}, \ldots, d_{n}\right)$, where $d_{1} \leq d_{2} \leq \ldots \leq d_{n}$. Let $b$ be a positive integer and let $n_{0} \geq 1$ be the number of vertices with degree at least $b$. Then for every positive integer $1 \leq k \leq n_{0}$,

$$
\rho_{b, k}^{\prime}(G) \geq \frac{\sum_{j=1}^{k}\left(b d_{j}+d_{j}^{2}\right)}{2 d_{n}}-m .
$$

Proof. Let $g$ be a $\rho_{b, k}^{\prime}(G)$-cover of $G$ and let $g(v) \geq b$ for $k$ distinct vertices $v$ in $B(G)=$ $\left\{v_{j_{1}}, \ldots, v_{j_{k}}\right\}$. Define $f: E(G) \rightarrow\{0,1\}$ by $f(e)=$ $(g(e)+1) / 2$ for each $e \in E(G)$. We have

$$
\sum_{e \in E(G)} f\left(N_{G}[e]\right)=\sum_{e=u v \in E(G)} \frac{g\left(N_{G}[e]\right)+\operatorname{deg}(u)+\operatorname{deg}(v)-1}{2} .
$$




$$
\sum_{e \in E(G)}\left(g\left(N_{G}[e]\right)+g(e)\right)=\sum_{v \in V} g(E(v)) \operatorname{deg}(v)
$$

and

$$
\sum_{e=u v \in E(G)}(\operatorname{deg}(u)+\operatorname{deg}(v))=\sum_{v \in V} \operatorname{deg}(v)^{2},
$$

by (1) it follows that

$$
\begin{aligned}
& \sum_{e \in E(G)} f\left(N_{G}[e]\right) \\
&= \frac{1}{2} \sum_{v \in V} \operatorname{deg}(v)(g(E(v))+\operatorname{deg}(v))-\frac{1}{2} \sum_{e \in E(G)} g(e)-\frac{m}{2} \\
& \geq \frac{1}{2} \sum_{v \in V \backslash\left\{v_{j_{1}}, \ldots, v_{j_{k}}\right\}} \operatorname{deg}(v)(g(E(v))+\operatorname{deg}(v))+ \\
& \frac{1}{2} \sum_{i=1}^{k}\left(b d_{j_{i}}+d_{j_{i}}^{2}\right)-\frac{1}{2} \rho_{b, k}^{\prime}(G)-\frac{m}{2} \\
& \geq \frac{1}{2} \sum_{i=1}^{k}\left(b d_{j_{i}}+d_{j_{i}}^{2}\right)-\frac{1}{2} \rho_{b, k}^{\prime}(G)-\frac{m}{2} \\
& \geq \frac{1}{2} \sum_{j=1}^{k}\left(b d_{j}+d_{j}^{2}\right)-\frac{1}{2} \rho_{b, k}^{\prime}(G)-\frac{m}{2} .
\end{aligned}
$$

On the other hand,

$$
\begin{aligned}
\sum_{e \in E(G)} f\left(N_{G}[e]\right) & =\sum_{v \in V} f(E(v)) \operatorname{deg}(v)-\sum_{e \in E(G)} f(e) \\
& \leq \sum_{v \in V} f(E(v)) d_{n}-\sum_{e \in E(G)} f(e) \\
& =d_{n}\left(2 \sum_{e \in E(G)} f(e)\right)-\sum_{e \in E(G)} f(e) \\
& =\left(2 d_{n}-1\right) \sum_{e \in E(G)} f(e) .
\end{aligned}
$$

By (2) and (3)

$$
\sum_{e \in E(G)} f(e) \geq \frac{\frac{1}{2} \sum_{j=1}^{k}\left(b d_{j}+d_{j}^{2}\right)-\frac{1}{2} \rho_{b, k}^{\prime}(G)-\frac{m}{2}}{2 d_{n}-1} .
$$

Since $g(E(G))=2 f(E(G))-m$, by (4)

$$
\rho_{b, k}^{\prime}(G)=\sum_{e \in E(G)} g(e) \geq
$$

$$
\frac{1}{2 d_{n}-1}\left(\sum_{j=1}^{k}\left(b d_{j}+d_{j}^{2}\right)-\rho_{b, k}^{\prime}(G)-m\right)-m .
$$

Thus,

$$
\rho_{b, k}^{\prime}(G) \geq \frac{\sum_{j=1}^{k}\left(b d_{j}+d_{j}^{2}\right)}{2 d_{n}}-m,
$$

as desired.

An immediate consequence of Theorem 2 is:

Corollary 3 For every $r$-regular graph $G$ of size $m$, $\rho_{b, k}^{\prime}(G) \geq \frac{k(b+r)}{2}-m$. Furthermore, the bound is sharp for $r$-regular graphs with $b=r$ and $k=n$.

Theorem 4 Let $G$ be a graph of order $n \geq 2$, size $m$, without isolated vertices, with minimum degree $\delta=\delta(G)$ and maximum degree $\Delta=\Delta(G)$. Let $b$ be a positive integer and $n_{0} \geq 1$ be the number of vertices with degree at least $b$. Then for each positive integer $1 \leq k \leq n_{0}$

$$
\begin{gathered}
\rho_{b, k}{ }^{\prime}(G) \geq \\
\frac{\left(\Delta^{2}+b^{2}\right) k-2(\Delta-\delta) m-(b-1)^{2} n-\left(\Delta^{2}-(b-1)^{2}\right) n_{0}}{2 \delta} .
\end{gathered}
$$

Furthermore, the bound is sharp for $n$-cycles when $b=2$ and $k=n$.

Proof. Let $B(G)=\{v \in V(G) \mid \operatorname{deg}(v) \geq b\}$ and let $f$ be a $\rho_{b, k}^{\prime}(G)$-cover. Since for each $v \in V^{+}, f(v) \geq b$, it follows that $|M \cap E(v)| \leq\left\lfloor\frac{\operatorname{deg}(v)-b}{2}\right\rfloor$. Thus

$$
(2 \delta-1)|M|
$$$$
\leq \sum_{e=u v \in M}(\operatorname{deg}(u)+\operatorname{deg}(v)-1)
$$$$
=-|M|+\sum_{e=u v \in M}(\operatorname{deg}(u)+\operatorname{deg}(v))
$$$$
=-|M|+\sum_{v \in V(G[M])}|M \cap E(v)| \operatorname{deg}(v)
$$$$
\leq-|M|+\sum_{v \in V^{+}}|M \cap E(v)| \operatorname{deg}(v)+\sum_{v \in V^{-}}|M \cap E(v)| \operatorname{deg}(v)
$$$$
\leq-|M|+\sum_{v \in V^{+}}\left\lfloor\frac{\operatorname{deg}(v)-b}{2}\right\rfloor \operatorname{deg}(v)+\sum_{v \in V^{-}} \operatorname{deg}(v)^{2}
$$$$
\leq-|M|+\sum_{v \in V^{+}} \frac{\operatorname{deg}(v)^{2}}{2}+\sum_{v \in V^{-}} \operatorname{deg}(v)^{2}-\frac{b}{2} \sum_{v \in V^{+}} \operatorname{deg}(v)
$$ 


$$
\begin{aligned}
& \leq-|M|+\sum_{v \in V} \frac{\operatorname{deg}(v)^{2}}{2}+\sum_{v \in V^{-}} \frac{\operatorname{deg}(v)^{2}}{2}-\frac{b^{2}}{2}\left|V^{+}\right| \\
& \leq-|M|+\Delta \sum_{v \in V} \frac{\operatorname{deg}(v)}{2}+\sum_{v \in V^{-} \cap B(G)} \frac{\operatorname{deg}(v)^{2}}{2}+ \\
& \sum_{v \in V^{-} \backslash B(G)} \frac{\operatorname{deg}(v)^{2}}{2}-\frac{b^{2}}{2}\left|V^{+}\right| \\
& \leq-|M|+\Delta m-\frac{b^{2}}{2} k+\frac{\Delta^{2}}{2}\left|V^{-} \cap B(G)\right|+\frac{(b-1)^{2}}{2}\left|V^{-} \backslash B(G)\right| \\
& \leq-|M|+\Delta m-\frac{b^{2}}{2} k+\frac{\Delta^{2}}{2}\left(n_{0}-k\right)+\frac{(b-1)^{2}}{2}\left(n-n_{0}\right)
\end{aligned}
$$

Hence,

$$
|M| \leq \frac{\Delta m}{2 \delta}+\frac{1}{4 \delta}\left((b-1)^{2} n+\left(\Delta^{2}-(b-1)^{2}\right) n_{0}-\left(\Delta^{2}+b^{2}\right) k\right) .
$$

Now (5) follows by the fact that $\rho_{b, k}^{\prime}(G)=m-2|M|$.

\section{An Upper Bound on SbkECN}

Bonato et al. in [11] posed the following conjecture on $\rho_{b}^{\prime}(G)$.

Conjecture 5 Let $b \geq 2$ be an integer. There is a positive integer $n_{b}$ so that for any graph $G$ of order $n \geq n_{b}$ with minimum degree $b$,

$$
\rho_{b}^{\prime}(G) \leq(b+1)(n-b-1) .
$$

Since $\rho_{b}^{\prime}\left(K_{b+1, n-b-1}\right)=(b+1)(n-b-1)$, the upper bound would be the best possible if the conjecture were true. They also proved that the conjecture is true for $b=2$. In this section we provide an upper bound for $\rho_{b, k}{ }^{\prime}(G)$, where $b=2$ and $1 \leq k \leq n$. The proof of the next theorem is essentially similar to the proof of Theorem 5 in [11].

Theorem 6 Let $G$ be a graph of order $n$, size $m$ and without isolated vertices. Let $n_{0}>0$ be the number of vertices with degree at least 2. Then for $n \geq 7$ and $1 \leq k \leq n_{0}$,

$$
\rho_{2, k}^{\prime}(G) \leq 2 n+k-9 .
$$

Proof. The proof is by induction on the size $m$ of $G$. By a tedious and so omitted argument, it follows that $\rho_{2, k}^{\prime}(G) \leq k+5$ if $n=7$. We may therefore assume that $n \geq 8$. Suppose that the theorem is true for all graphs $G$ without isolated vertices and size less than $m$. Let $G$ be a graph of order $n \geq 8$, size $m$ and without isolated vertices. We will prove that $\rho_{2, k}^{\prime}(G) \leq 2 n+k-9$ for each $1 \leq k \leq n_{0}$. We consider four cases.

Case 1. $\delta(G)=1$.

Let $u$ be a vertex of degree 1 and $v \in N(u)$. First suppose $\operatorname{deg}(v)=1$. Then the induced subgraph $G[u, v]$ is $K_{2}$. It is straightforward to verify that $\rho_{2, k}^{\prime} \leq 2 n+k-9$ when $n=8$. Hence, we may assume that $n \geq 9$. Let $G^{\prime}=G-u v$. Then $G^{\prime}$ is a graph of order $n-2 \geq 7$, size $m-1$ and without isolated vertices. By the inductive hypothesis, $\rho_{2, k}^{\prime}\left(G^{\prime}\right) \leq 2(n-2)+k-9=2 n+k-13$. Let $f$ be a $\rho_{2, k}^{\prime} \quad\left(G^{\prime}\right)$-cover. Define $g: E(G) \rightarrow\{-1,1\}$ by $g(u v)=-1$ and $g(e)=f(e)$ if $e \in E(G)-u v$. Obviously, $g$ is a S2kEC and so

$$
\rho_{2, k}^{\prime}(G) \leq \rho_{2, k}^{\prime}\left(G^{\prime}\right)-1 \leq 2 n+k-14<2 n+k-9 .
$$

Now suppose $\operatorname{deg}(v) \geq 2$. Consider two subcases.

Subcase $1.1 \operatorname{deg}(v) \geq 3$.

By the inductive hypothesis on $G-u, \rho_{2, k}^{\prime}(G-u)$ $\leq 2(n-1)+k-9=2 n+k-11$. Let $f$ be a $\rho_{2, k}^{\prime}$ $(G-u)$-cover and define $g: E(G) \rightarrow\{-1,1\}$ by $g(u v)=1 \quad$ and $\quad g(e)=f(e) \quad$ if $\quad e \in E(G)-u v$. Obviously, $g$ is a S2kEC and so

$$
\rho_{2, k}^{\prime}(G) \leq \rho_{2, k}^{\prime}\left(G^{\prime}\right)+1 \leq 2 n+k-10<2 n+k-9 .
$$

Subcase $1.2 \operatorname{deg}(v)=2$.

Let $w \in N(v)-\{u\}$. If $k=1$, then define $g: E(G) \rightarrow\{-1,1\}$ by $g(u v)=g(v w)=1$ and $g(e)=$ -1 if $e \in E(G) \backslash\{u v, v w\}$. Obviously, $g$ is a $\mathrm{S} 2 \mathrm{kEC}$ of $G$ and we have

$$
\rho_{2, k}^{\prime}(G) \leq g(E(G))=4-m \leq 2 n+k-9 .
$$

Let $k \geq 2$. It follows that $n_{0} \geq 2$. By the inductive hypothesis on $G-\{u\}, \quad \rho_{2, k-1}^{\prime}(G-\{u\}) \leq 2(n-1)+$ $(k-1)-9=2 n+k-12$. L e t $f \quad$ b e a $\rho_{2, k-1}^{\prime}$ $(G-\{u\}) \quad$-cover. Define $g: E(G) \rightarrow\{-1,1\} \quad$ by $g(u v)=g(v w)=1$ and $g(e)=f(e)$ if $e \in E(G) \backslash$ $\{u v, v w\}$. Obviously, $g$ is a $\mathrm{S} 2 \mathrm{kEC}$ and so

$$
\rho_{2, k}^{\prime}(G) \leq \rho_{2, k-1}^{\prime}(G-\{u\})+3 \leq 2 n+k-9 .
$$


Case 2. $\delta(G)=2$.

Let $w$ be a vertex of degree 2 and $N(w)=\{u, v\}$. Consider two subcases.

Subcase 2.1 $u v \notin E(G)$. Let $G^{\prime}$ be the graph obtained from $G-\{w\}$ by adding an edge $u v$. Then $G^{\prime}$ has order $n-1$, size $m-1$ and at least $k-1$ vertices with degree at least 2 . By the inductive hypothesis,

$$
\rho_{(k-1), 2}^{\prime}\left(G^{\prime}\right) \leq 2(n-1)+(k-1)-9=2 n+k-12 .
$$

Let $f$ be a $\rho_{2, k}^{\prime}\left(G^{\prime}\right)$-cover. Define $g: E(G) \rightarrow$ $\{-1,1\}$ by $g(u w)=g(v w)=1$ and $g(e)=f(e)$ if $e \in E(G) \backslash\{u v, v w\}$. Obviously, $g$ is a S2kEC and so

$$
\rho_{2, k}^{\prime}(G) \leq g(E(G)) \leq f\left(E\left(G^{\prime}\right)\right)+3 \leq 2 n+k-9 .
$$

Subcase 2.2 $u v \in E(G)$. First let both $u$ and $v$ have degree 2. Then the induced subgraph $G[\{u, v, w\}]$ is an isolated triangle. If $1 \leq k \leq 3$, then define $f: E(G) \rightarrow\{-1,1\}$ by

$$
f(u v)=f(v w)=f(u w)=1 \text { and } f(e)=-1 \text { otherwise. }
$$

Then

$$
\rho_{2, k}^{\prime}(G) \leq f(E(G))=6-m \leq 2 n+k-9 .
$$

Now suppose that $k \geq 4$. It is not hard to show that $\rho_{2, k}^{\prime}(G) \leq 2 n+k-9$ when $n=8$ or 9 . Hence, we may assume that $n \geq 10$. Let $G^{\prime}=G \backslash\{u, v, w\}$. Then $G^{\prime}$ is a graph of order $n-3 \geq 7$, size $m-3$ and has at least $k-3$ vertices with degree at least 2 . By the inductive hypothesis, $\rho_{2,(k-3)}^{\prime}\left(G^{\prime}\right) \leq 2(n-3)+(k-3)-9=2 n+$ $k-18$. Let $f$ be a $\rho_{2,(k-3)}^{\prime}\left(G^{\prime}\right)$-cover. Define $g: E(G) \rightarrow\{-1,1\}$ by

$g(u v)=g(v w)=g(u w)=1$ and $g(e)=f(e)$ if $e \in E\left(G^{\prime}\right)$.

Obviously, $g$ is a $\mathrm{S} 2 \mathrm{kEC}$ of $G$ and

$$
\rho_{2, k}^{\prime}(G)=g(E(G)) \leq f\left(E\left(G^{\prime}\right)\right)+3 \leq(2 n+k-18)+3 .
$$

Now let $\min \{\operatorname{deg}(u), \operatorname{deg}(v)\} \geq 3$. If $k=1$, define $g: E(G) \rightarrow\{-1,1\}$ by $g(u w)=g(v w)=1$ and $g(e)=$ -1 otherwise. Obviously, $g$ is a S2kEC and so

$$
\rho_{2, k}^{\prime}(G) \leq g(E(G))=4-m<2 n+k-9 .
$$

If $k \geq 2$, then $G^{\prime}=G-\{w\}$ is a graph of order $n-1$, size $m-2$ and has at least $k-1$ vertices with degree at least 2. By the inductive hypothesis, we have that $\rho_{2,(k-1)}^{\prime}\left(G^{\prime}\right) \leq 2 n+k-12$. Let $f$ be a $\rho_{2,(k-1)}^{\prime}\left(G^{\prime}\right)$-cover. We can obtain a S2kEC $g$ of $G$ by assigning $g(e)=1$ for each $e \in E(G) \backslash E\left(G^{\prime}\right)$ and $g(e)=f(e)$ for each $e \in E\left(G^{\prime}\right)$. Then we have

$g(E(G))=f\left(E\left(G^{\prime}\right)\right)+2=\rho_{2,(k-1)}^{\prime}\left(G^{\prime}\right)+2<2 n+k-9$.

Hence, $\rho_{2, k}^{\prime}(G)<2 n+k-9$, as desired.

Finally, assume $\min \{\operatorname{deg}(u), \operatorname{deg}(v)\}=2$. Let without loss of generality $\operatorname{deg}(u)=2$. If $1 \leq k \leq 2$, define $g: E(G) \rightarrow\{-1,1\}$ by $g(u w)=g(v w)=g(u v)=1$ and $g(e)=-1$ otherwise. Obviously, $g$ is a S2kEC and so

$$
\rho_{2, k}^{\prime}(G) \leq g(E(G))=6-m \leq 2 n+k-9 .
$$

If $k \geq 3$, then $G^{\prime}=G-\{w\}$ is a graph of order $n-1$, size $m-2$ and has at least $k-2$ vertices with degree at least 2. By the inductive hypothesis,

$$
\rho_{2,(k-2)}^{\prime}\left(G^{\prime}\right) \leq 2(n-1)+(k-2)-9=2 n+k-13 .
$$

Let $f$ be a $\rho_{2,(k-2)}^{\prime}\left(G^{\prime}\right)$-cover. Define $g: E(G) \rightarrow$ $\{-1,1\}$ by

$$
\begin{gathered}
g(u v)=g(v w)=g(u w)=1 \text { and } g(e)=f(e) \\
\text { if } e \in E\left(G^{\prime}\right) \backslash\{u v\} .
\end{gathered}
$$

Obviously, $g$ is a $\mathrm{S} 2 \mathrm{kEC}$ of $G$ and

$$
\rho_{2, k}^{\prime}(G) \leq g(E(G))=f\left(E\left(G^{\prime}\right)\right)+4 \leq 2 n+k-9 .
$$

Case 3. $\delta(G)=3$.

Let $w$ be a vertex with degree 3 . If $k=1$, define $g: E(G) \rightarrow\{-1,1\} \quad$ by $g(u w)=1 \quad$ if $u \in N(w)$ and $g(e)=-1$ otherwise. Obviously, $g$ is a S2kEC and so

$$
\rho_{2, k}^{\prime}(G) \leq g(E(G))=6-m<2 n+k-9 .
$$

If $k \geq 2$, then $G^{\prime}=G-\{w\}$ is a graph of order $n-1$, size $m-3$ and has at least $k-1$ vertices with degree at least 2. By the inductive hypothesis, we have that $\rho_{2,(k-1)}^{\prime}\left(G^{\prime}\right) \leq 2 n+k-12$. Let $f$ be a $\rho_{2,(k-1)}^{\prime}$ $\left(G^{\prime}\right)$-cover. We can obtain a S2kEC $g$ of $G$ by assigning $g(e)=1$ for each $e \in E(G) \backslash E\left(G^{\prime}\right)$ and $g(e)=f(e)$ for each $e \in E\left(G^{\prime}\right)$. Then we have

$$
g(E(G))=f\left(E\left(G^{\prime}\right)\right)+3=\rho_{2,(k-1)}^{\prime}\left(G^{\prime}\right)+3 \leq 2 n+k-9 .
$$

Hence, $\rho_{2, k}^{\prime}(G) \leq 2 n+k-9$, as desired.

Case 4. $\delta(G) \geq 4$. 
Then $G$ has an even cycle by Theorem 6 . Let $C=\left(v_{1}, v_{2}, \ldots, v_{s}\right)$ be an even cycle in $G$. Obviously, $G^{\prime}=G-E(C)$ is a graph of order $n$, size $m-|E(C)|$ and has at least $k$ vertices with degree at least 2 . By the inductive hypothesis, $\rho_{2, k}^{\prime}\left(G^{\prime}\right) \leq 2 n+k-9$. Let $f$ be a $\rho_{2, k}^{\prime}\left(G^{\prime}\right)$-cover. Let $v_{s+1}=v_{1}$ and define $g: E(G) \rightarrow$ $\{-1,1\}$ by

$$
\begin{gathered}
g\left(v_{i} v_{i+1}\right)=(-1)^{i} \text { if } i=1, \ldots, s \text { and } g(e)=f(e) \text { for } \\
e \in E(G) \backslash E(C) .
\end{gathered}
$$

Obviously, $g$ is a S2kEC and hence $\rho_{2, k}^{\prime}(G)=$ $\rho_{2, k}^{\prime}\left(G^{\prime}\right) \leq 2 n+k-9$. This completes the proof.

\section{Conclusions}

In this paper we initiated the study of the signed $(b, k)$-edge cover numbers for graphs, generalizing the signed star domination numbers, the signed star $k$ domination numbers and the signed $b$-edge cover numbers in graphs. The first lower bound obtained in this paper for the signed $(b, k)$-edge cover number concludes the existing lower bounds for the other three parameters. Our upper bound for the signed $(b, k)$-edge cover number also implies the existing upper bound for the signed $b$-edge cover number. Finally, Theorem 6 inspires us to generalize Conjecture 5 .

Conjecture 7 Let $b \geq 3$ be an integer. There is a positive integer $n_{b}$ so that for any graph $G$ of order $n \geq n_{b}$ with $n_{0} \geq 1$ vertices of degree at least $b$, and for any integer $1 \leq k \leq n_{0}, \quad \rho_{b, k}^{\prime}(G) \leq b n+k-(b+1)^{2}$.

\section{References}

[1] D. König, "Über trennende knotenpunkte in graphen (nebst anwendungen auf determinanten und matrizen)," Acta Litterarum ac Scientiarum Regiae Universitatis Hungaricae Francisco-Josephinae, Sectio Scientiarum Mathematicarum [Szeged], Vol. 6, pp. 155-179, 19321934.

[2] R. Rado, "Studien zur kombinatorik," Mathematische Zeitschrift, German, Vol. 36, pp. 424-470, 1933.

[3] T. Gallai, "Über extreme Punkt-und Kantenmengen (German),” Ann. Univ. Sci. Budapest. Eötvös Sect. Math., Vol. 2, pp. 133-138, 1959.

[4] R. Z. Norman and M. O. Rabin, "An algorithm for a minimum cover of a graph," Proceedings of the American Mathematical Society, Vol. 10, pp. 315-319, 1959.

[5] A. Schrijver, "Combinatorial optimization: Polyhedra and Efficiency," Springer, Berlin, 2004.

[6] D. B. West, "Introduction to graph theory," Prentice- Hall, Inc., 2000.

[7] B. Xu, "On signed edge domination numbers of graphs," Discrete Mathematics, Vol. 239, pp. 179-189, 2001.

[8] C. Wang, "The signed star domination numbers of the Cartesian product," Discrete Applied Mathematics, Vol. 155, pp. 1497-1505, 2007.

[9] B. Xu, "Note on edge domination numbers of graphs," Discrete Mathematics, Vol. 294, pp. 311-316, 2005.

[10] B. Xu, "Two classes of edge domination in graphs," Discrete Applied Mathematics, Vol. 154, pp. 1541-1546, 2006.

[11] R. Saei and S. M. Sheikholeslami, "Signed star $k$-subdomination numbers in graph," Discrete Applied Mathematics, Vol. 156, pp. 3066-3070, 2008.

[12] A. Bonato, K. Cameron, and C. Wang, "Signed b-edge covers of graphs (manuscript)". 